\title{
ON THE EXPERIENCE OF TENSELESS TIME
}

\section{NATHAN OAKLANDER}

\section{UNIVERSITY OF MICHIGAN-FLINT}

\begin{abstract}
Defending the tenseless theory of time requires dealing adequately with the experience of temporal becoming. The issue centers on whether the defender of tenseless time can provide an adequate analysis of the presence of experience and the appropriateness of certain of our attitudes toward future and past events. By responding to a recent article, 'Passage and the Presence of Experience', by H. Scott Hestevold, I shall attempt to show that adequate analysis of tenseless time is possible.
\end{abstract}

\section{$\mathbf{T}$}

he status of temporal becoming, temporal passage, or the transitory aspect of time is a paradigmatic metaphysical problem. It involves a prima facie conflict between reason and experience. The experience in question involves the passage of time; the "perception" of events flowing from the future into the present and from the present into the past. This experience is reflected in statements such as 'I can't wait until the basketball season comes around again', 'Hurray, I am finally graduating', and 'Thank goodness the exam is over!' When we rationally reflect upon these statements and wonder what reality must be like in order for them to be true we find logical difficulties, such as McTaggart's paradox, emerge. Faced with this conflict the goal of the metaphysician is to provide an ontology of time that fits the experience in question and is logically consistent. Broadly speaking, two theories of time have taken up the challenge to realize that goal: the tensed and the tenseless theories. According to the tenseless view, the logical problems surrounding temporal becoming are real and can only be avoided by recognizing, in Donald C. Williams' words, 'the myth of passage' [15]. According to the tensed view, the experience of passage and the presence of experience are real and can only be accounted for by accepting the tenses as reflecting basic ontological distinctions. ${ }^{1}$

The debate between proponents of the two camps has been fought on 
several different fronts. Until the early 1980's the question of translatability was of central importance. If tensed discourse could be defined without loss of meaning into tenseless discourse then it was argued the tenses lacked ontological significance. More recently, defenders of the so-called "new tenseless theory of time" have sought to demonstrate that the necessity of tensed discourse is compatible with time being tenseless. ${ }^{2}$ In order to do this successfully it is necessary for the detenser to deal adequately with the experience of temporal becoming. The issue centers on whether the defender of tenseless time can provide an adequate analysis of the presence of experience and the appropriateness of certain of our attitudes toward future and past events. In a recent article, 'Passage and the Presence of Experience', H. Scott Hestevold argues that the tenseless theory of time cannot account for our experience of time [3]. In what follows, I shall attempt to show his objections to the tenseless theory can be overcome, and an adequate analysis of tenseless time is possible.

\section{The Presence of Experience}

According to the tenseless theory of time there are no basic ontological differences between past, present and future events. All events exist tenselessly in the network of earlier, later and simultaneity, temporal relations. If, however, all events exist tenselessly, then how can the detenser explain our knowledge that a certain experience, say a headache, is [presently] occurring? How can the detenser explain the fact that experiences can be known to be present? According to Hestevold, no explanation is possible because

The claim that experiences, essentially, can be known to be present implies that there cannot occur an experience which occurs only tenselessly; experiences cannot be mere tenseless occurrences on the B series! ([3]: 543)

The reasoning underlying the implication in the above passage is open to two interpretations. First, since none of the terms of the B-series (the series of events generated by the earlier-later relation) are intrinsically present, no experienced events on the B-series can be known to be present. Second, if the detenser defines the presence of an experience in terms of its occurring at a certain date, or its being simultaneous with some temporal item, then it follows all experiences are (tenselessly) present. In that case, however, the detenser cannot explain the knowledge we possess of which experiences are happening now. For if all our experiences exist tenselessly at the moment they do, what is the explanation for the phenomenological fact that certain of those experiences are known to be occurring now while others are not known to be occurring now, or are even known not to be occurring now?

I think the detenser has a reasonably good response to that question. It begins with the truism that whenever we are aware of an object (or have an 
experience) we are conscious of being aware of that object (or of having that experience). Thus, one aspect of our knowledge of the present is grounded in the consciousness of our experiences at the time they are occurring. If we combine that thesis with the claim by Thomas Reid that "consciousness ... is an immediate knowledge of the present' ([10]: 359) we arrive at the required result: For an individual, every experience he or she is conscious of is one known to be present. Of course, Hestevold may ask, 'Doesn't this claim from Reid posit something that really is present? If you need that claim to finish off your argument, haven't I made my point?' Not necessarily, because Reid's reference to 'the present' can be understood to designate the cross-section of experiences that are simultaneous with one's consciousness of them. There is nothing more, ontologically speaking, to the presence of experience than our being conscious of our experiences when they are happening.

To this explanation of the presence of experience it may be objected that merely being (tenselessly) conscious of an experience when it is (tenselessly) occurring does not give knowledge of which experiences are [presently] occurring. But I do not think this objection can be sustained, for the knowledge we seek can be explained tenselessly, and the argument to the contrary is a non sequitur.

According to a detenser, if $I$ am conscious at $t_{1}$ of an experience that occurs tenselessly at $t_{1}$, and if as a matter of tenseless fact it is $t_{1}$, then I know the experience is present. Of course, tensers use the same antecedent to infer that detensers cannot know which experiences are present, but such an inference is based on a misinterpretation of the tenseless view. The tenseless view gives rise to several different images. One is that of experiences in the B-series existing "eternally" or totally outside of time. Another views experiences as existing sempiternally or at every time; and still another views experiences from a point of view outside of time, looking down at all experiences and events and seeing them as parts of a never changing present. Each of these images falsifies the detenser's view in a fundamental way. On the tenseless theory, experiences and events are not eternal or sempiternal, and they do not all exist at once, totum simul. Rather, experiences, like our consciousness of them, exist in time, in succession, one after another. We are in time and, therefore, conscious of our experiences from a temporal point of view. The significance of this last point can be clarified by means of a spatial analogy. We are in space, and so experience things from a spatial point of view. I am here, hence distant from some places and near others. Accordingly, the answer to the question, 'Which things are existing here?' depends on the place at which the question is asked. Similarly, the answer to the question, 'Which events are existing now?' depends on the time at which the question is asked. Right now, as I look at the clock on my desk it is 10:00 am, September 11, 1992 and so the experience of my looking at the clock (of which I am conscious) is known by me to be present. There is no 
need to suppose there is any special property of events that are present, or objects that are here, that enable us to know which events are present or which objects are here. Admittedly, if we were somehow outside of time, and so nontemporally conscious of all our experiences (as God might be of the history of the world), then no experience could be known to be present to the exclusion of others. But our consciousness of experiences, like the experiences themselves, are in time and at any given time we can know what experiences are present simply by being conscious of them as opposed to remembering or anticipating them.

To all this Hestevold makes the following reply. If, at $t_{1}$, I record the presence of my experience of say, an excruciating toothache, by telling the dentist, 'I am now in pain' then on the tenseless theory that means ' $t_{1}$ is tenselessly occurring, and my excruciating pain is (tenselessly) occurring at $\mathrm{t}_{1}$ '. However, that judgment is true at any time, and so would not be sufficient to convey to the dentist the requisite information, namely, that I am in pain now. In order to convey that information the tensed fact that my pain is now occurring is indispensable.

Once again, Hestevold's argument is a non sequitur. It proves that the tenseless sentence [ ${ }^{\prime} t_{1}$ is tenselessly occurring, and my excruciating pain is (tenselessly) occurring at $\left.t_{1}{ }^{\prime}\right]$ does not have the same meaning as the tensed sentence ('I am now in pain'), but it does not prove that the two sentences are used to describe different states of affairs; one describing a tenseless fact and the other describing a tensed fact (cf. Williams [14]). Admittedly, the tensed sentence conveys more information than the tenseless one, but it does not follow that it does so because of the reality of tense. The dentist who hears the tensed sentence token 'I am now in pain' knows that I am using that sentence to describe a state of affairs existing simultaneous with my utterance, and so he or she administers the anaesthetic. Whereas if I uttered the tenseless sentence the dentist would not know that I needed relief now unless he knew what time it was. Thus, the two sentences do not convey the same information and so do not have the same meaning. Nevertheless, it does not follow that they do not describe the same state of affairs and, more generally the indispensability of tensed discourse does not imply the indispensability of tensed facts.

Before leaving the topic of the presence of experience, I want to consider another phenomenological datum that allegedly supports the tensed theory. George Schlesinger has defended the tensed theory by appealing to the experience of the NOW as 'the point in time at which an individual who is temporally extended is alive, real or Exists with a capital E' ([12]: 23). More recently he claimed 'our attitude toward the present may be described as regarding it as distinct from every other temporal position, for while the future is yet to be born and the past is rapidly fading, the present is palpably real' ([11]: 427). I suggest we can make sense of Schlesinger's phenomenological claims without countenancing transitory temporal properties. Again, 
a spatial analogy may help. I am here, and so experience space differently from the way I would if I were outside of space. I can know what goes on in distant places, and given causal laws, I can affect what goes on elsewhere less surely, and reasonably regard what goes on there as less important because it affects my life much less. Similarly, I am now (at this time), hence those events that are at temporally distant times are less affected by me and have less affect upon me, than those which are in the present. Thus, I may reasonably regard what is happening now as being more important, or more real, and that is the only (harmless) sense in which the present is "palpably real" or Exists with a capital E; the reality of tense has nothing to do with it.

\section{Our Attitudes Toward the Future and the Past}

Another argument intended to demonstrate that the tenseless theory of time cannot be squared with our experience is based on the claim that dread and relief are inexplicable attitudes on the tenseless theory. Hestevold explains the reasoning underlying this claim in the following passage:

On Monday I dread the painful tooth extraction scheduled for Tuesday, and on Wednesday I am relieved that the extraction is over. Dread on Monday and relief on Wednesday are appropriate attitudes to have toward the Tuesday tooth extraction. If ST [the static theory] is correct, however, then dread and relief are never appropriate since there are no future and past events toward which to direct them! That Wednesday follows the day of the tooth extraction is a tenseless fact which is true before, during and after the extraction. Thus it is as appropriate to feel relief that Wednesday follows Tuesday before or during the extraction as it is to feel such relief on Wednesday. But this is absurd. ... Hence, TT [the tensed theory] must be adopted to make sense of the appropriateness of our attitudes toward the future and the past ([3]: 544-545).

Hestevold's point is that since the fact, Wednesday is later than Tuesday, is a fact that exists before Wednesday, if that fact is what explains relief, then it is just as sensible to feel relief on Monday or Tuesday for a painful experience that is taking place on Tuesday as it is to feel relief on Wednesday for the same painful experience.

The mistake in this argument is the assumption that the tenseless fact that renders relief appropriate exists before, during and after the extraction. On the tenseless view, the fact in question does not exist before, after or during the extraction. The pain exists before the relief, and the experience of the relief exists after the cessation of pain, but the fact that the pain occurs before Wednesday (or that the relief occurs after the pain) does not exist in time at all. Thus, while it is "always" true to assert that "Wednesday follows Tuesday' it does not follow that Wednesday's following Tuesday always exists and so Hestevold ought not conclude that relief is justified before the 
pain or during it. To think that it is justified is to confuse tenseless facts with sempiternal things.

Further evidence that Hestevold does make that confusion occurs when he says,

After all, on Wednesday, there is a sense in which the extraction is not over; on Wednesday, the extraction is "eternally" and tenselessly occurring on Tuesday ([3]: 545).

Again, this way of viewing the matter is fraught with difficulties. To say that an extraction is tenselessly occurring on Tuesday $\left(t_{2}\right)$ is to say, assuming that time is relational, that the extraction is simultaneous with each member of the set of simultaneous events that constitutes $t_{2}$. That fact, however, does not exist on Tuesday, or on Wednesday, or on any other day; it is eternal. But to say that an event's occurring at a certain time is a n eternal fact, does not imply that the event in some sense is always occurring, although looked at from an external Godlike perspective it may appear as if this is so. But from the inside, and in reality, our painful experiences are (hopefully) shortlived, and as they are succeeded by more pleasant experiences my awareness of the painful ones become a mere memory. Indeed it is just this succession of different psychological attitudes toward the same event (first anticipation, then consciousness, then memory) that gives rise to the impression of time's flow, and it is that impression that provides the basis for our different attitudes toward the future and the past.

Accepting all this, a critic may wonder why treating facts as outside of time helps to resolve Hestevold's problem. If it is always true that the dread occurs before the painful experience, and the fact of the dread occurring before the pain never changes, why should I be happy now that the toothache is over? Of course, if the painful experience will occur, but is not yet occurring, that is, if it will move from the future to the present with the passage of time, then, so the critic alleges, we can easily understand an attitude or feeling of dread. We cannot understand that attitude on the tenseless theory, where all events exist and nothing really moves through time at all. In short, the tenseless theory never explains why dread is "appropriate" before a bad event rather than after or during it.

One way of responding to this objection is to question the premise upon which it is based, namely, the assumption that the feeling of dread is appropriate when the dreaded event is in the future. Perhaps we should say dread is an appropriate attitude to take before an unwelcome event in that it is a rational attitude to take. However, it might plausibly be argued that dread often is not rational if it does not make us more efficient in meeting the problems we face. Dread of a dentist's visit does nothing but make one's life miserable before the visit, and it may even stop us from keeping our appointments. It serves in no way to direct one's actions, since the visit is necessary for good health. So, although it is natural enough, perhaps, at 
least for a certain sort of personality, to dread certain sorts of events, it is not clear it is appropriate in the sense of being rational.

Maybe it is in general useful to dread bad events because dread in general motivates us to prepare for or avoid such events in ways that we would not employ if we did not experience dread. So it is easy to see how dread might evolve biologically. But, like many biologically evolved defence systems, this one often actually does harm, preventing us from acting efficiently, and so must often be controlled or suppressed if one is to behave more reasonably. Dread before the event is functional (when dread is functional at all) because one can still do something about it. The same feeling after the event is never functional, so never appropriate. Thus, our attitude toward dread is like the attitude we have toward preparing oneself, say, for an exam - it makes sense before but not after the exam because preparation affects the outcome. Similarly, if dread spurs on preparation it might have survival value and thus be appropriate before the event, but not after. In other words, the causal efficacy of dread, and the direction of causality in time are what explain its appropriateness before, but not after, the event. ${ }^{5}$ Since that explanation works perfectly well for a detenser, I conclude neither the presence of experience nor our attitudes toward the past, present or future pose insurmountable difficulties for an adequate analysis of tenseless time. ${ }^{6}$

\section{REFERENCES}

[1] Michelle Beer, 'Temporal Indexicals and the Passage of Time', The Philosophical Quarterly 38 (1988): 158-164.

[2] Brian Garrett, “'Thank Goodness That's Over” Revisited', The Philosophical Quarterly 38 (1988): 201-205.

[3] H. Scott Hestevold, 'Passage and the Presence of Experience', Philosophy and Phenomenological Research 50 (1990): 537-552.

[4] Delmas Kiernan-Lewis, "Not Over Yet: Prior's "Thank Goodness" Argument', Philosophy 66 (1991): 256-258.

[5] Murray MacBeath, 'Mellor's Emeritus Headache', Ratio 25 (1983): 81-88.

[6] D. Hugh Mellor, Real Time (Cambridge: Cambridge University Press, 1981).

[7] L. Nathan Oaklander, Temporal Relations and Temporal Becoming: A Defense of a Russellian Theory of Time (Lanham, MD.: University Press of America, 1984).

[8] _ _A Defence of the New Tenseless Theory of Time', The Philosophical Quarterly 41 (1991): 26-38.

[9] __ 'Thank Goodness It's Over', Philosophy 67 (1992): 256-258.

[10] Thomas Reid, Essays on the Intellectual Powers of Man (Cambridge, Mass.: MIT Press, 1969).

[11] George Schlesinger, 'E Pur Si Muove', The Philosophical Quarterly 41 (1991): 426-441.

[12] Aspects of Time (Indianapolis: Hackett Publishing Company, 1980). 
[13] Quentin Smith, Language and Time (New York: Oxford University Press, 1993).

[14] Clifford Williams, 'The Date-Analysis of Tensed Sentences', The Australasian Journal of Philosophy 70 (1992): 198-203.

[15] Donald C. Williams, 'The Myth of Passage', The Journal of Philosophy 48 (1951): 457-472.

\section{ENDNOTES}

${ }^{1}$ The most elaborate defense of the tensed theory of time is found in Quentin Smith [14].

2Proponents of the new tenseless theory of time include Michelle Beer [1], D. H. Mellor [5], and L. Nathan Oaklander [7], [8].

${ }^{3}$ Recent discussions of this type of argument are found in Brian Garrett [2], Delmas Kiernan-Lewis [4], and L. Nathan Oaklander [8].

${ }^{4}$ Also, of course, the word 'dread' contains 'before the event' in its meaning or usage-after the event one may regret or rue it, or look back on it with horror, but one cannot dread it. But looking back on it with horror is close enough to the feeling of dread so we can get away from mere grammar here and ask why such a feeling of horror is not appropriate after it is over. If one holds that dread is appropriate even when it is dysfunctional, merely because the event really is awful, then looking back on it with horror would also be appropriate.

5I wish to thank the editor and an anonymous referee for the Journal of Philosophical Research for their helpful comments, and the University of Michigan-Flint for an award that partially funded research for this paper. 